

\title{
DE LO INDUSTRIAL A LO CONVIVENCIAL
}

\begin{abstract}
From a qualitative review of grade papers, and dialogues with the students who prepared them, in this paper I review, within the academic space of degree project of the tadeísta industrial design program a confluence of agendas and interests of actors with dissimilar ratings on the evaluation criteria and about what design "must be". I perceive a latent tension between two trends: the industrial one, based on a fixed identity and where rigorous approaches of design prevail; and the convivial one, based on multiple identifications, where the excitement and chaos are possibilities to concrete people of flesh and blood. The guidelines of the first are dogmatic (from Greek, dokein: 'teach', 'indoctrinate') and are useful to foster in the students a culture of be followers of the teachers; those of the second one are zetetics (from Greek, Zetein: 'search', 'inquire'), by means of these ones the students can learn even from the spontaneity and error.
\end{abstract}

Keywords: Dogmatic Design, Zetetic Design, Project Degree, Evaluation Criteria, Conviviality.

\section{Introducción}

El Homo convivium, a diferencia del individuo moderno, recobra y despliega sus cualidades de convivencia, para restaurar sus capacidades de diseñar herramientas convivenciales; esto es bienes e instituciones de cualquier índole mediante la participación y el encuentro, desde las relaciones y los vínculos entre personas que conviven (cf. Mattié, 2013, s.p.).

Diseño este texto, con eso en mente para presentarlo en sociedad en Cali. Comprendo que al emplear la primera persona, abandono cualquier aspiración científica para aproximarme a las veleidades ensayísticas y las travesuras poéticas. Algo que encuentro apropiado para reflexionar sobre el ritual académico universitario del proyecto de grado, tal cual lo vivimos en el Programa de Diseño Industrial tadeísta. Es esa instancia de validación final que para cada estudiante requiere transponer las fronteras del pregrado y entrar al país del profesionalismo. Incluyo este proceso entre lo que Arnold Van Gennep denominó "ritos de paso": pues está circunscrito al protocolo transicional entre el fin de la adolescencia estudiantil del pregrado, y la inclusión, que culminar con éxito tal empresa supone, en la 'tribu' de los diseñadores industriales titulados (cf. Van Gennep en 'Rite of passage', 2013, n.p.). 
En el contexto bogotano del Programa de Diseño Industrial de la Universidad Jorge Tadeo Lozano hemos procurado combinar la validación de lo diseñado, con la comunicación, por quien lo diseñó del proceso investigativo creativo que siguió para diseñarlo ( $c f$. Friedman 2013: s.p.). Formativamente es el lugar donde el estudiante prueba unas destrezas intelectuales y unas habilidades prácticas que depura en una asignatura inicial de Investigación para Proyecto de Grado (IPG) donde plantea su anteproyecto desde tres ejercicios ( $c f$. Gutiérrez, 2012, pp. 73-74):

1. Una autobiografía, para considerar cómo el diseño industrial ha incidido en su aprendizaje, aun antes de asistir a la Universidad; la aspiración es que encuentre los patrones que pormenorizan su actuación, conforme haya experimentado sus asignaturas para hallar su perfil.

2. Los hallazgos o invenciones suscitados por la autobiografía, soportan una etapa de ensayo donde explora posibles modos de interrelacionar los elementos de su proyecto que pueden ser ejes, variables, categorías, etc., de acuerdo al método por el que opte, esto es por el que, en la mayoría de los casos, los profesores optamos en su lugar.

3. Como corolario del ensayo es formulado un anteproyecto cuya consumación acontece en la siguiente asignatura de Curso para Proyecto de Grado (CPG), la cual como estamos fascinados con los finales, es por constante histórica en los tiempos recientes, para muchos estudiantes el momento en que "ahora sí de verdad y en serio" es elaborado el proyecto de grado.

A menudo lo generado en la asignatura inicial de investigación es desdeñado por los propios estudiantes, entre otras cosas por el desinterés al respecto de algunos de los tres profesores que para cada grupo acompañan la asignatura final.

Desde 2008 cuando fue institucionalizado, la intención tras el actual modelo, es que con la asistencia de los profesores, grupos de 15 estudiantes, integren equipos de diseño entre pares. O intenten hacerlo, pues hay varios bloqueos para el proceso: primero, la inclinación mayoritaria de los profesores a instruir y de los estudiantes a seguir; segundo, la dificultad que entrañan y lo poco desarrolladas que están (aunque esté de moda aplicar por doquier el adjetivo "participativo") las didácticas que fomenten una coautoría y una coproducción en diseño bastante complejas, tanto de enseñar, para quienes crecimos y aún laboramos separados en el medio académico, como de aprender, para quienes son aún evaluados en solitario. 
Durante ambas asignaturas, los estudiantes exploran cursos proyectuales para los cuales modelan soluciones mediante cuatro recursos:

1. Sistema de registro (documento para consignar la estructuración del pensamiento práctico orientado a los diversos públicos y actores involucrados en el proyecto).

2. Cronograma dinámico (un instrumento de reflexión permanente sobre el empleo del tiempo como insumo proyectual, cuyo dinamismo en la práctica es limitado debido a las restricciones de un calendario con el mismo número de semanas para todos y por el lugar común del llenado de casillas que Excel y otros programas computacionales suministran).

3. Medios de socialización (personalización del modo propio de exponer el proyecto; algo en que la libertad de elección queda a reducida a lo que de tipografía y software maneje cada quien en el tiempo asignado para presentar, esto es 15 minutos).

4. Criterios de evaluación (enunciados argumentales para dar cuenta de la manera en que cada quien valora el diseño y aplica su capacidad de juicio al proyecto).

Me aparto aquí de los requisitos planteados para los estudiantes para preguntarme por el modo en que los profesores los valoramos, no a los estudiantes, sino a los requisitos: ¿cuáles son pues nuestros criterios de evaluación para valorar los criterios de evaluación de los estudiantes? Mi respuesta es "aquellos en los que creemos" y a raíz de ella percibo una polaridad que nos cobija a todos los profesores en uno $\mathrm{u}$ otro aspecto generando diversas tensiones.

\section{Discusión/reflexión}

Deseo precisar que desconozco la realidad como 'Es', por cuanto asumo que sólo la puedo conocer, como todos, por las implicaciones que tiene para mí estar inmerso en ella. En consecuencia me expreso mediante mis creencias y mis aspiraciones. Basado en ellas pienso que vivimos en una época en la que ante el diseño industrial se alza la alternativa del diseño convivencial, no porque las teorías, autores y prácticas que soportan al segundo sean novedosas, sino por el momento histórico que han ganado.

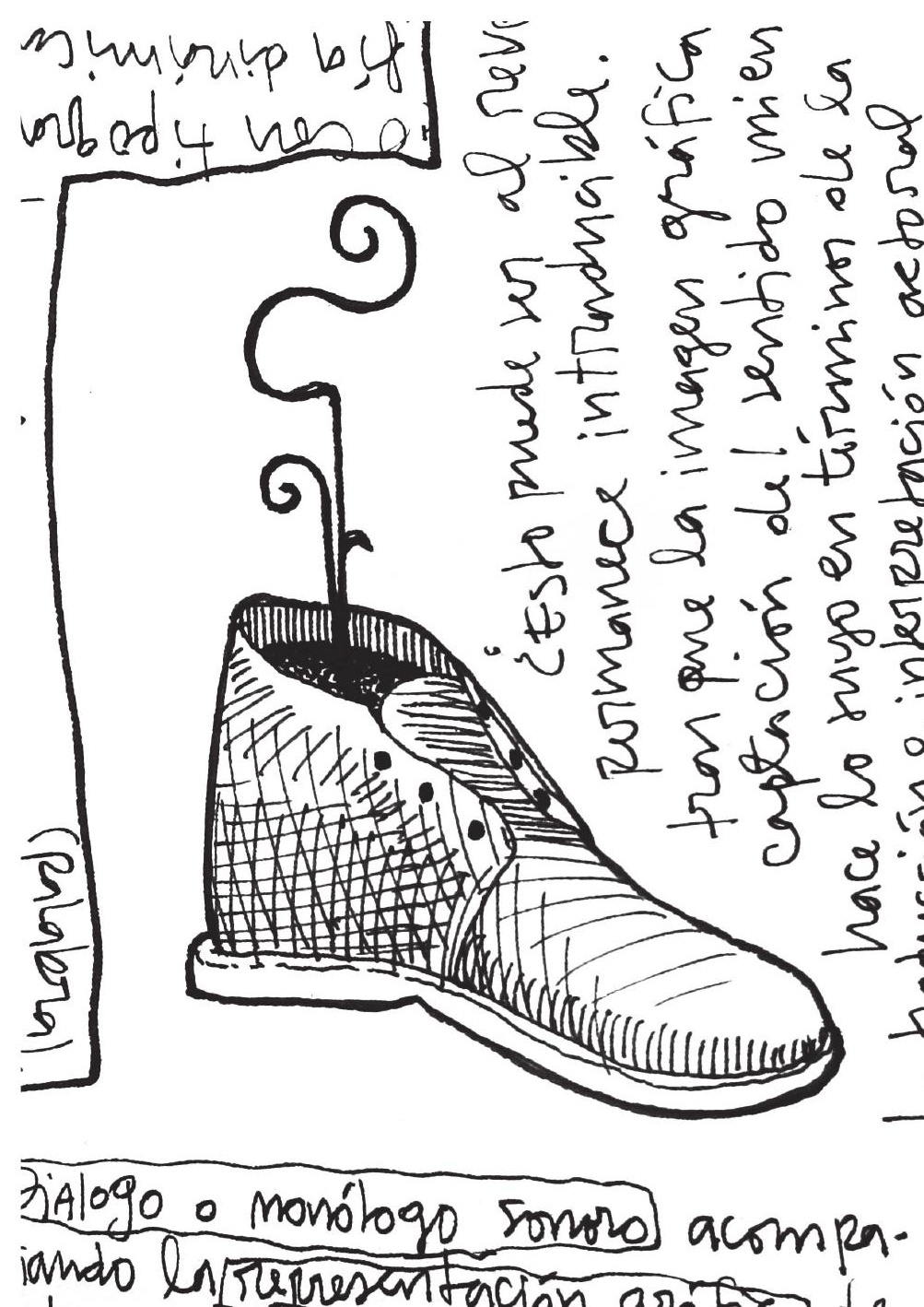


Lo industrial en el diseño es para mí el paradigma vigente, lo convivencial, el paradigma al cual la transición apunta. Entre lo industrial y lo convivencial hay tensiones mediante las que intentaré explicar cómo comprendo ambos extremos: la principal la encuentro entre el diseño dogmático y el diseño zetético, como los observo en las prácticas.

Permítaseme aquí problematizar el sustantivo 'diseño', a partir de los adjetivos con que lo designo, y a través de adjetivos para designar a estos adjetivos. De esta suerte podré explicar la primera tensión entre un diseño industrial dogmático, y un diseño convivencial zetético.

El más conocido para los eventuales públicos de este trabajo será el adjetivo 'dogmático' procedente del griego dokéin (enseñar, adoctrinar) y relacionado con el dogma como verdad establecida y principio organizador de las prácticas. La educación y el diseño son dogmáticos cuando prevalece como norma la instrucción del profesor sobre el alumno, o del diseñador sobre el usuario, con una función directiva de los primeros, ante cuyas preguntas ordenadoras a los segundos sólo les queda responder. Los profesores dogmáticos, de diseño u otro saber, están interesados en la esencia, en la identidad como algo fijo y único, en la disciplina, y en métodos generales para resolver los proyectos.

En contraste, está el adjetivo zetético, del griego zetéin (buscar, inquirir) y en algunos textos en español y portugués aparece escrito con 'c' y no con 'z'; éste está relacionado con la zétesis como duda informativa y principio reformador de las prácticas. La educación y el diseño son zetéticos cuando la pauta vinculante es el énfasis en el aspecto de la pregunta como condición informativa y especulativa de la investigación y base del aprendizaje de los estudiantes, o de todos los seres humanos como diseñadores cotidianos (frente a los diseñadores profesionales). Los profesores zetéticos, de diseño u otro saber, estamos interesados en la emergencia, en la identificación como algo cambiante, y en la indisciplina que permite recrear mitos en torno a los proyectos.

Allí donde los dogmáticos regulan, los zetéticos emancipan. La mentalidad de los primeros es de sístole, de rigor y contracción, la de los segundos es de diástole, de elasticidad y distensión. Por supuesto, no hay dogmáticos ni zetéticos totales y el asunto no se trata de ser dogmático o zetético, sino de alternar el matiz según momentos particulares de situaciones particulares. El inconveniente que encuentro es que en la institucionalidad industrial el dogmatismo aparece, por descarte, como elección única.

Retomo mi reflexión sobre el proyecto de grado de diseño industrial tadeísta, para el cual con los años, los profesores del Programa establecimos tres horizontes amplios para culminar los proyectos: Objeto, Interacción y Contexto, los cuáles transformamos en rutas exploratorias amplias dentro del presente plan de estudio; en dicho marco, el trabajo de grado puede ser adelantado a lo largo de cada una de las rutas en tres posibles modalidades con los inevitables proyectos de carácter híbrido entre ellas, a saber: 
1. Producto (desarrollo de producto industrial).

2. Enfoque empresarial (para la consolidación desde la inserción del diseño en la estrategia productiva y ejemplificando desde proyectos productivos puntuales, ya una empresa, ya una estrategia empresarial de diseño industrial) y;

3. Investigación: para hacer una contribución documental a los avances dentro de la investigación de diseño en el contexto tadeísta (cf. Gutiérrez, 2012, pp. 73-74).

Como coordinador, desde el año 2009, del área de proyecto de grado, donde se insertan las asignaturas acerca de las cuales discurro, he decantado las impresiones que aquí comparto; y mi experiencia se ha enriquecido con la revisión documental que efectuamos junto con el profesor Camilo Andrés Angulo Valenzuela durante una

de las etapas de la investigación

que adelantamos actualmente $\mathrm{y}$ culminaremos en marzo de 2014

para caracterizar los proyectos de grado de diseño industrial tadeísta entre 2009 y 2011, y situarlos frente a diversos enfoques cartográficos del diseño contemporáneo.

Como la inmensa mayoría de las presentaciones ante los jurados de quienes elaboraron los proyectos no están registradas en video, ni subsisten los modelos materiales que las soportaron, ni hay acceso a la interacción dialógica que aconteció en cada situación puntual, decidimos evaluar en principio los documentos de grado que los estudiantes entregan para archivo en biblioteca.

Sobre estos cabe señalar, en gracia de discusión, que no son los que determinan las calificaciones finales, sino las más de las veces un requisito que, por cómo hemos manejado las cosas a la fecha, cumplen los estudiantes con desgano y cuando ya saben que han aprobado la sustentación del proyecto ante jurados y, además, la nota con la cual lo han hecho.

El otro aspecto es entrevistar a losactores implicados en dicha socialización, estudiantes (hoy profesionales), y profesores para conocer sus recuerdos e impresiones. Aquí hemos encontrado a la fecha que el asunto de la sustentación es una reflexión aparentemente en fuera de lugar para muchos profesores, que generalmente cae en el olvido pasado el suceso. 
En cuanto a los estudiantes, buena parte declaran haber experimentado desagrado con su proyecto de grado (algo en la mayoría de los casos directamente proporcional a la nota obtenida o al número de veces que repitieron el curso). No obstante, aún algunos entre quienes han obtenido buenas notas señalan que hallaron poco futuro en un proyecto de grado que resulta estéril por haber sido elaborado al gusto de los profesores y no al de ellos.

Como los documentos, según señalé toman cuerpo una vez pasada la sustentación, tampoco son muchos el tiempo o el trabajo que los profesores tomamos para revisarlos, es cual si profesores y estudiantes los asumiésemos como un mal necesario. De ahí y en la mayoría de los casos, los vacíos en la redacción, la repetición de fuentes, la pobreza y el desorden en asuntos de citación; sin mediar reflexión profunda, muchos de esos documentos tienen, para las nuevas generaciones de estudiantes que cursen las asignaturas de proyecto de grado, mucho menos valor del que podrían tener si fuera el gusto y no la obligación, el motivo para realizarlos.

Poco depararán la creatividad domada, y las aulas donde los profesores llamamos diálogo a nuestro monólogo, algo que puede acontecer también en eventos académicos mientras lo dogmático y zetético estén desequilibrados, es más mientras la idea convivencial del diseño zetético sea desconocida para la mayoría de los profesores y estudiantes de diseño.

Lo más importante sobre el ritual del proyecto de grado, cabe preguntar aquí ¿es confirmar lo esperado, o esperar lo inesperado? La disciplina industrial causa lo primero, la indisciplina convivencial puede reportar lo segundo.

Con Klaus Krippendorff, rechazo para el diseño, el calificativo de 'disciplina' que evoca castigos para los desobedientes y demanda sumisión irreflexiva ante la norma, como acontecería en una fuerza armada mercenaria. En una vena más convivencial, y aunque él no use este término, para Krippendorff el diseño es un 'campo', según la metáfora sociológica, el cual podemos abonar para sembrar y cultivar muchas variedades de plantas, incluso dando lugar a que crezcan algunas malezas (cf. 1998, p. 2). El concepto de campo resulta, así contemplado, más zetético, y el de disciplina, más dogmático.

En virtud del desplazamiento semántico metonímico, los significados de un mismo término cambian, algo que un pensamiento demasiado disciplinado, y por ende muy dogmático nos impide entender. Pensemos por ejemplo, en la evolución de los enfoques sobre cibernética y teoría de sistemas.

Inicialmente, el término designaba formas industriales de comprensión de los fenómenos mediante el control. Pero convivencialmente, al pasar de las rigurosas disciplinas de las ciencias, a los campos flexibles de los asuntos sociales, su eje fue desplazado del objeto al sujeto (la polisemia o multiplicidad de significados del mismo término es algo que el dogmatismo dificulta considerar). 
Por ello hay discrepancias entre: quienes cuando escuchan los términos "teoría de sistemas y cibernética" piensan dogmáticamente en enfoques mecánicos para predecir y controlar, por ejemplo, la calidad en las líneas productivas, esto es: investigadores de objetos o sistemas observados; y quienes, al escuchar las mismas palabras, pensamos zetéticamente en el modo caprichoso y complejo en el que, pese a las regulaciones más extremas, se comportan, no sólo los seres humanos que trabajan en el aseguramiento de la calidad en las líneas productivas, cuyo desempeño pretenden controlar y predecir los expertos dogmáticos, sino aún dichos expertos y hasta a nosotros mismos al estudiar a todos los demás “como siendo estudiados por nosotros", esto es investigadores de sujetos o sistemas observantes (cf. Van den Merwe, 2010, p. 8).

En un enfoque priman los valores técnicos, en el otro los humanos con sus valores. En el antiguo esquema griego de academia, la hipótesis de partida es que la evidencia de tu comprensión de algo es que puedas explicarlo; eso distinguiría al estudiante académico que sería más zetético así observado. A la inversa en la tradición judeocristiana, la dogmática es fundamental (¿o "funda mental”?), toda vez que lo importante es aceptar y creer una doctrina aunque no se la comprenda bien, como hacen los discípulos que identifican, sin poder explicarlos, los misterios de lo que ha sido o está siendo practicado conforme a la doctrina del maestro.

Bajo un marco zetético, un estudiante académico puede teorizar su ejercicio, allí donde un discípulo dogmatizado sólo se entrena para practicar lo que cree (o lo que fue empujado a creer). Ocasionalmente, como recuerda Ivar Holm, el estudiante académico comprende materias teóricas con poca o ninguna consideración por su practicidad, allí donde el estudiante discípulo entrena para convertirse en practicante de sus creencias. De tal modo, un discípulo es educado en algo que quizá ni entiende, ni puede explicar, pero debe practicar. El estudiante académico, en cambio, diseña teorías propias para entender y explicar (cf. 2006, p. 65).

Nuestras preguntas respecto a los problemas más importantes en diseño, y a las formas idóneas de resolverlos, están usualmente cargadas de valor. Por eso las respondemos con frecuencia de modo subjetivo. La objetividad completa, según explica Bryan Lawson (1997, p. 126) supone apartar nuestras emociones de los proyectos, algo que como humanos difícilmente podemos hacer; así, a más comprometidos con lo diseñado más personales nuestro juicios al respecto. El diseño convivencial nos permite notar que no trabajamos tanto para enfrentar el cómo y el porqué de lo que es, cuanto para buscar lo que puede, podría y debería ser; diseñamos para participar en la prescripción y la creación de los futuros en que querríamos que otros habitaran con nosotros, algo que merece escrutinio ético y también moral (cf. 1997, p. 126). 
Al darle esencias a los términos se me antoja que la tensión dogma/zétesis encubre el antiguo choque entre lo apolíneo y lo dionisiaco, o entre lo masculino y lo femenino, o entre la razón y la emoción (ubicada a la base de las inteligencias de segunda para los más dogmáticos).

Como fuere, en el proyecto de grado tadeísta el énfasis está en el aprendizaje validado en del manejo de una estructura, más que de un tema; y ello acontece en una relación entre estudiantes y profesores donde a través de la interacción continua (antagónica o empática) consciente o inconscientemente inculcamos a nuestros alumnos nuestros valores cardinales ( $c f$. Holm, 2006, p. 66).

La práctica del diseño convivencial requiere "abandonar el papel de experto o especialista, algo que para muchos diseñadores casi equivale a perder el sentido de sí mismos" (Nelson, 1994). Migrar de la era de los expertos dogmáticos a la era de la coordinación de las experiencias de los actores convivenciales requiere trabajar pedagógicamente nociones no sólo del co-diseño, sino de la coautoría.

La relación profesor/alumno, sin importar cuán objetiva aparente ser, está basada en el diseño como la aplicación de un sistema de creencias que reviste expresiones particulares según quiénes la escenifiquen; a la larga es la coincidencia o discrepancia en la adhesión a unos valores (generalmente los de los profesores evaluadores), más que el entendimiento de una teoría primordial (cf. Holm 2006, pp. 65-66), lo que en buena parte de los casos determina, hoy por hoy, la aprobación del proyecto de grado de diseño industrial tadeísta por parte de los estudiantes.

\section{¿Método?}

Al dogma de preguntar por el deber ser del proyecto de grado, lo acompaño con la zétesis de buscarle el sentido del proyecto de profesión. Eso creo es lo que se define actualmente en la arena argumental del espacio del proyecto de grado dentro del Programa de diseño industrial tadeísta, en un encuentro de agendas e intereses de profesores y estudiantes cuya multiplicidad de bases axiológicas involucra tantas percepciones sobre el "deber ser" del ejercicio como participantes haya en él.

Muchos colegas a quienes respeto y aprecio consideran desde sus valores el diseño como un ejercicio de control regulado por normas científicas; yo lo asumo como una actividad con fuerte componente político donde casi toda acción requiere concertar, disentir (cf. Holm, 2006, p. 285) y escuchar la voz de cada actor.

Lo anterior me lleva académicamente a impugnar uno de los rasgos más opresivos del enfoque moderno e industrial: la censura al derecho de cada quien a dar cuenta de sí mismo, evidenciada en la imposición de emplear la tercera persona en el texto académico. La instauración de un diseño convivencial y zetético comienza por recuperar la voz del sujeto concreto en el texto académico, especialmente en uno sobre diseño. Pensando en eso diseñé mi ponencia. 
No nos llamemos a engaño, si es por criterios de evaluación, en diseño siempre dependen en algún grado de quien evalúa, por eso medir el diseño es tan complicado, los rumbos de los proyectos están tan cargados de valores como gran parte de la ruta seguida para solucionarlos, sin importar cuan técnicas sea algunas pruebas y mediciones efectuadas en el entretanto, nuestra percepción general al respecto es subjetiva y es que "Lo que puede parecer importante para un profesor o un estudiante puede no serlo para otros. Por ende, las formulaciones enteramente objetivas en los problemas de diseño son un imposible” (Lawson, 1997, pp. 120-121).

Debido a sus valores y creencias, los profesores y estudiantes de diseño buscan mediante una retórica deliberativa argumentos de base para las decisiones que van a tomar (cf. 2006, p. 337). Que tal cosa acontezca en la mayoría de los casos no implica que muchos la noten, y menos aún lo acepten.

La crítica convivencial al diseño industrial está soportada en el "Juicio de diseño", como una forma de deliberación que requiere interpretaciones inalcanzables con el modo científico que obtiene verdades en contextos controlables y predecibles. (cf. Nelson, 1994).

El diseño convivencial lo sugirió en 1970 Ivan Illich para cuestionar el monopolio del modo de producción industrial que transforma a las personas en materia prima, personas-herramientas, para elaborar más herramientas (cf. 1978, s.p.).

El dogmatismo en el diseño industrial, y aquí lo indeseable no está en el dogma, sino en el 'ismo' (en tanto uso dogmático del dogma), propicia que de los Programas de diseño, y ese para el que yo trabajo aún es ejemplo de ello, se gradúen profesionales capaces de buscar trabajo, e incluso en generarlo, pero no de encontrarle sentido a esas búsquedas y emprendimientos. Para subsanar eso en un enfoque convivencial el acento técnico industrial, es sustituido por el ético (cf. Illich, 1978, s.p.).

Desdeunaproximación zetética, la pregunta no es tanto cuánto aprendió el estudiante, aprendiz de diseñador, de lo que sus profesores le enseñamos, sino cuánto y cómo lo apoyamos en que consiga desplegar todo su potencial para aprender y aplicar por sí mismo.

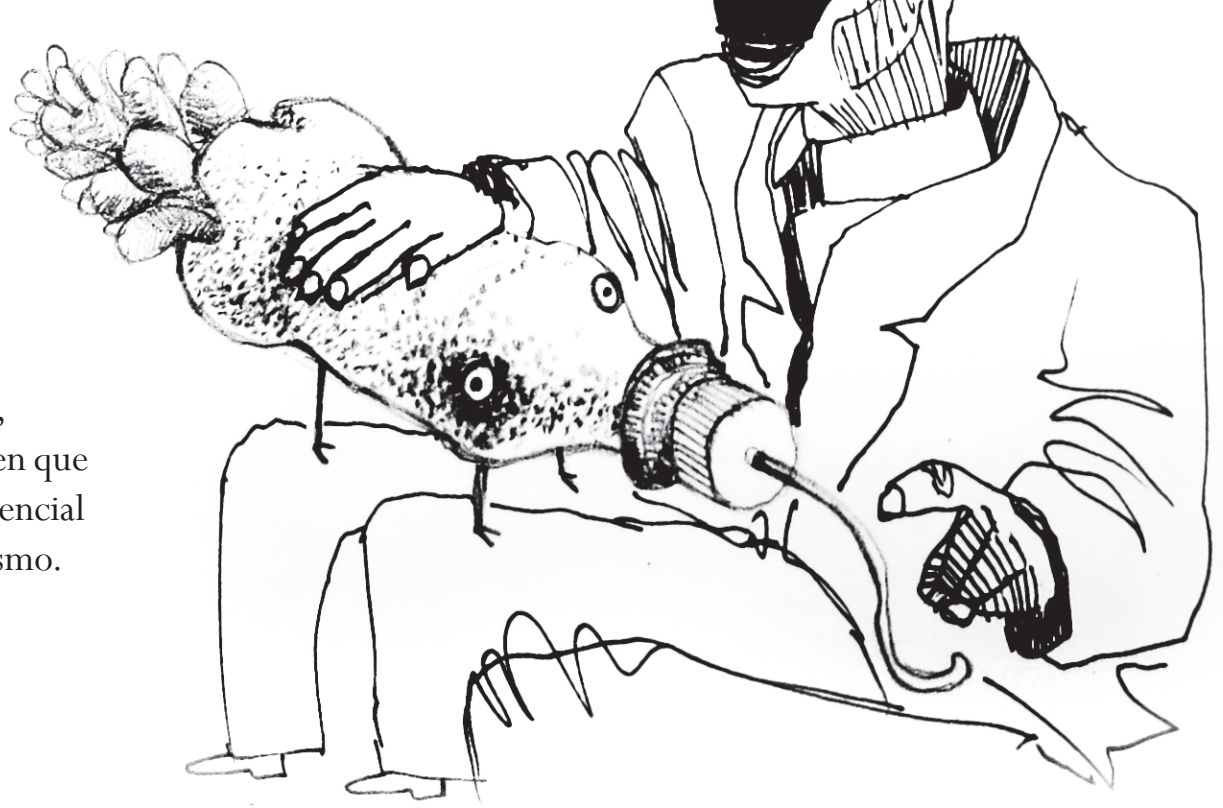




\section{Conclusiones ¿o continuaciones?}

Cuando señalo que para diseñar la sociedad colombiana, podríamos pensar menos en la rentabilidad industrial y más en la interacción convivencial, acepto lo panfletario de la afirmación, pero es que somos las personas las que diseñamos, aún sin haber materializado, al dar sentido, con nuestra intencionalidad que convierte palos y piedras en herramientas. Es ese sentido que atribuimos el que convierte el objeto en artefacto: el 'facto' que nos implica como 'factores' o elaboradores.

La aproximación convivencial no sólo lleva al diseñador críticamente a revisar su rol profesional, sino a suspender la discriminación del diseñador aficionado (esto es de todo humano cuando planea la futura configuración de su entorno); lo convivencial implicaría abrir las fronteras del diseño a las multitudes y abandonar una visión mercantil del sistema de las profesiones merced a la cual el diseño es algo inalcanzable para las personas comunes que sólo llevan a cabo los grandes maestros iniciados y sus seguidores.

Illich diagnosticó para nuestras sociedades que, de no tomar el sendero de la convivencialidad, sucumbirían en una productividad hipertrofiada que jamás conseguiría satisfacer "las necesidades creadas y multiplicadas por la envidia”. (1978, s.p.).

El mejor panorama que imagino es el de programas de diseño industrial desprogramados, o en constante labor de desprogramación creativa, cuyos integrantes cuestionen la lógica de la producción masiva y del pensamiento estereotipado. Programas donde la creatividad del diseñador esté destinada al propósito de que los demás puedan ejercer cada vez más la suya. Aunque el técnico configure los detalles funcionales de los artefactos, es el poeta que vive en cada diseñador el que reivindica el poder de dar sentido al mundo, y ese poeta, quizás no le viene de su condición de diseñador, sino de ser humano.

Los artefactos industriales imponen a quienes los usan el sentido que les dio el fabricante o un diseñador profesional dogmático; los artefactos convivenciales permiten que cada persona que interactúe con ellos los emplee para dotar de sentido su mundo.

Mi parecer es que eso sucede aún poco dentro del proyecto de grado como lo manejamos en el Programa de Diseño Industrial de la Universidad Jorge Tadeo Lozano, eso aunque yo haya sido todos estos años un defensor del modelo. Muchas veces cuando como profesores de diseño cedemos a la tentación del dogma y decimos "ese es un buen estudiante" lo que insinuamos es que "ese diseña como le enseñé" o "como yo hago o digo" o "como yo le dejo diseñar". 
El inconveniente no está en el modelo, sino en nosotros que aún no conseguimos que cada estudiante elabore un proyecto ajustado a la proyección que quiere dar de sí mismo; y quién práctica el diseño sin entenderlo, aunque lo haga bien en lo técnico ¿cómo podría reflexionar al respecto en lo ético? Presumo que quien no ha podido encontrar el sentido de su profesión, queda predispuesto a impedir que otros lo hagan.

Imagino un mundo donde para elaborar cualquier artefacto la gente requiriera un permiso firmado por un funcionario del ministerio de diseño, y se me antoja la más inhumana pesadilla.

En el diseño convivencial hay que buscar otros tipos de novedades, a veces en lo primitivo y pasado de moda. Aunque luzca pobre o tacaño soy un enamorado de usar cosas viejas; y más precisamente porque según consignó Illich, en la sociedad de consumo somos discriminados cuanto mayor edad tienen los objetos que utilizamos (cf. 1978, s.p.).

No quiero un futuro dogmático para el cual hemos sido proyectados aburridos con lo que tenemos y enfermos por lo que no tenemos, quiero un presente donde podamos investigar por placer, sin que la especialización nos separe de las demás personas.

Poco importa lo que los profesores hagamos, los dilemas y oportunidades que trae la vida no aparecen dócilmente para ser manejados mediante las categorías de un disciplinado currículo universitario. Educar convivencialmente implica, para mí, facilitar que los estudiantes logren integrar constelaciones de conocimiento para cada ocasión y eso implica no un experto disciplinado sino un juicioso indisciplinado que haga valer su experiencia al servicio de otros; en la reactiva, dogmática e industrial estrategia de la solución de problemas, el proyecto de grado es algo que muchos estudiantes sortean a disgusto; en la proactiva y zetética dinámica convivencial el resorte para llevar a cabo dicho proyecto sería idealmente la intención de aproximarse a los estados de vida más deseados por cada quién ( $c f$. Nelson, 1994).

La actitud zetético-convivencial para diseñar nos impulsa a cuestionar el saber dogmatizado, y a buscar los obstáculos epistemológicos del conocimiento validado. Lo dogmática-industrial siempre produce obstáculos epistemológicos, y al elegir ahorra y degenera variables ( $c f$. Warat 1980, p. 112). De un tiempo acá he comenzado a pensar y a escribir sobre un diseño del sur convivencial, en el cual mediante la zétesis sean cuestionadas la obligatoriedad del diseño dogmatizado del norte como requisito y el desprecio a quienes fallan en conseguirlo. Un diseño del sur para aprovechar la experiencia desperdiciada de aquello a lo que somos cegados por la especialización y por ejercer un control que nos controla. 
En veinte años de docencia he encontrado muchos estudiantes que han depurado su competencia técnica y otros tantos que no; sin embargo siempre he tenido la persistente sensación de que los educadores prestamos poca atención a la parte convivencial presente en todos ellos, en cada futuro diseñador o diseñadora, esa a partir de la cual puede desplegar sus capacidades para generar relaciones personales y sociales (cf. Mattié, 2013, s.p.).

Es con eso en la mente y en el corazón que he escrito este texto para socializarlo hoy martes 24 de septiembre de 2013, durante este evento de Diseño en Sociedad 9, en esta ciudad de Cali, como muestra del convivencial afecto que motiva las relaciones que hay entre los agregados de estudiantes y profesores en vía de convertirse en comunidades de diseño industrial (y, ojalá, en un futuro, en comunidades de diseño convivencial), de las Universidades del Valle y Jorge Tadeo Lozano.

Atribuya cada quien a estas letras, el sentido que a bien tenga, no aspiro a haber dicho nada novedoso, pero sí a aportar a un diálogo con la esperanza de que consigamos al fin escuchar esas voces para las cuales la industrialización, la estandarización y el profesionalismo dogmático nos han tapado los oídos.

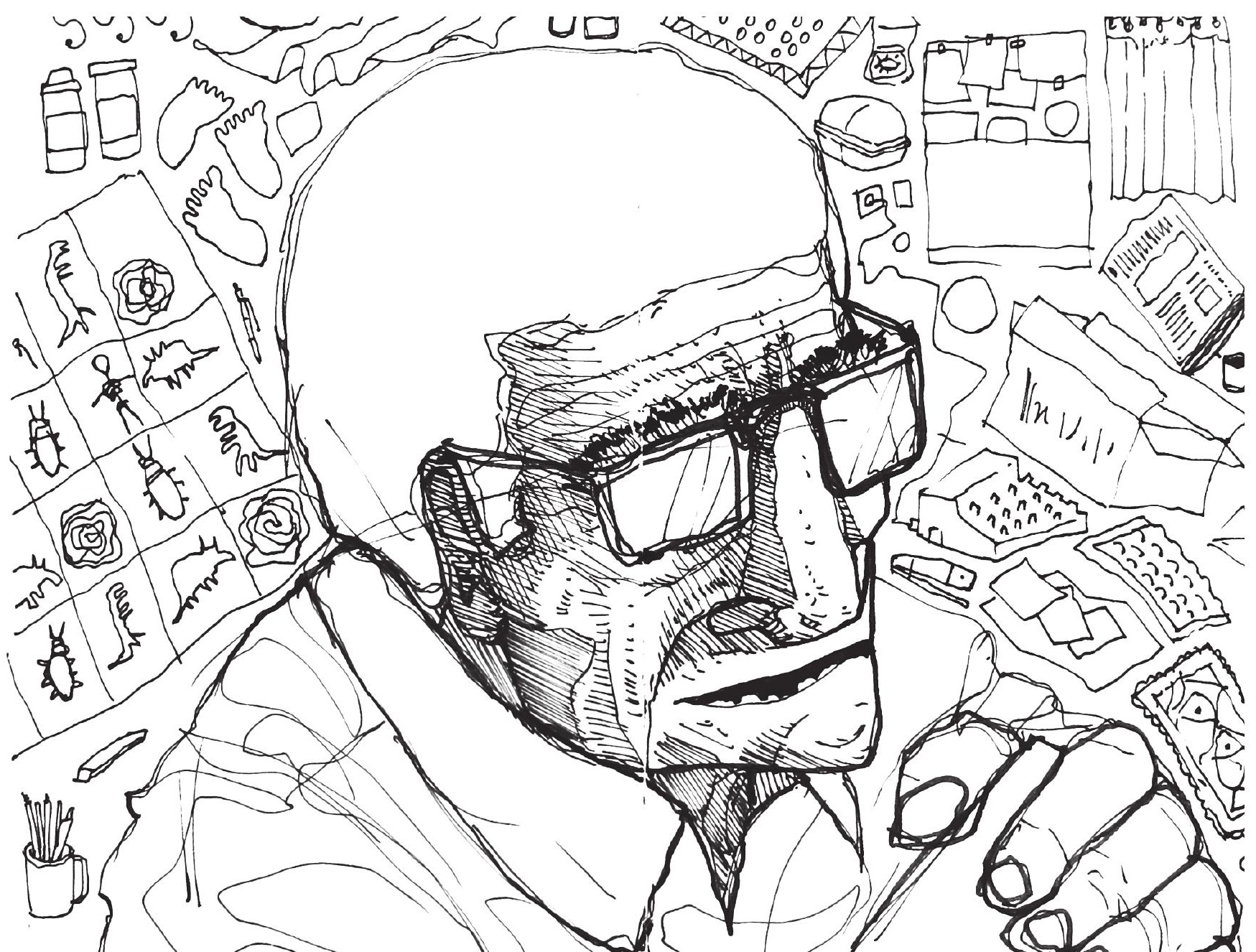




\section{Referencias}

Friedman, K. (2013). "Writing Research Requires Words" correo electrónico al grupo PHDDESIGN@JISCMAIL.AC.UK, sábado, marzo 9 en https: / /www.jiscmail.ac.uk/cgi-bin/ webadmin? $2=$ ind $1303 \& \mathrm{~L}=$ phd-design $\& \mathrm{~F}=\& \mathrm{~S}=\& \mathrm{P}=27483$

Gutiérrez, A. (2012). Diseño y tecnología: aproximaciones de género en estudiantes de diseño industrial en Revista S\&T, 10(22), Memorias: 5o Encuentro Internacional de Investigación en Diseño Diseño + 2012, pp. 69-79 ICESI, Colombia [en línea].

Holm, I. (2006). Ideas and beliefs in architecture and industrial design: How attitudes, orientations, and underlying assumptions shape the built environment. Oslo: AHO, The Oslo School of Architecture and Design.

Illich, I. (1978). La convivencialidad. en http: / habitat.aq.upm.es/boletin/n26/aiill.html

Krippendorff, K. (1998). A Field for Growing Doctorates in Design? en R. Buchanan, et al. (Eds.) Doctoral Education in Design (1998): Proceedings of the Ohio State Conference. Pittsburgh, PA: School of Design, Carnegie Mellon University, 1999. Pp. 207-224, versión en línea, con paginación independiente en ScholarlyCommons. http: / / repository.upenn.edu/asc_papers / 241

Lawson, B. (1997). How designers think:The design process demystified. Oxford: Elsevier/Architectural.

Mattié, M. (2013). “La persona convivencial” en Instituto Simone Weil, publicado por Sylvia María Valls texto en línea sin paginación. Recuperado de

http:/ / www.institutosimoneweil.net/index.php?option= ${ }_{\text {com_ }}$ content $\&$ view $=$ article $\&$ id $=423$ :la-persona-convivencial $\&$ catid $=48:$ mailer-mattie $\&$ Itemid $=68$

Nelson, H. G. (January 01, 1994). The Necessity of Being “Un-Disciplined” and “Out-of-Control” Design Action and Systems Thinking. Performance Improvement Quarterly, 7, 3, pp. 22-29. versión en línea, con paginación independiente en http:/ / www.advanceddesign.org/necessity.html

Rite of passage. (2013, June 21). InWikipedia, The Free Encyclopedia. English version. Ver http: / / en.wikipedia.org/w/index.php?title=Rite_of_passage\&oldid=560961997

Van der Merwe, J. (2010). A Natural Death Is Announced, en Design Issues, Summer 2010, Vol. 26, No. 3, pp. 6-17 (C) 2010 Massachusetts Institute of Technology Posted Online July 22, 2010. doi:10.1162/DESI_a_00025

Warat, L. A. (2010). “Cuestiones dogmáticas y cetéticas más allá de Tercio Sampaio” en Seqüência: estudos jurídicos e políticos; V. 01 n. 01 (originalmente 1980); 109-113; Fundação José Arthur Boiteux disponible en http://periodicos.ufsc.br/index.php/sequencia/article/view/17342

Recibido: abril 15 / Aprobado: junio 1 de 2014 